\title{
Using a Competence Model to Aggregate Learning Knowledge Objects
}

\author{
Amal Zouaq ${ }^{1}$, Roger Nkambou ${ }^{2}$, and Claude Frasson ${ }^{1}$ \\ 'University of Montreal, CP 6128, Succ. Centre-Ville, Montreal, QC, H3C3J7 \\ ${ }^{2}$ University of Quebec in Montreal, CP 8888, Succ. Centre-Ville, Montreal, QC, H3C3P8 \\ \{zouaq,frasson\}@iro.umontreal.ca \\ nkambou.roger@uqam.ca
}

\begin{abstract}
Competence-based learning models have great importance for learning resources: they constitute a meaningful structure for just-in-time and just-enough learning. In this paper, we present an ontology-based competence model that allows the on-the-fly generation of Learning Knowledge Objects (LKOs). The automatic aggregation process relies on knowledge objects and ontologies created through text mining and natural language processing. It is guided by instructional theories encoded declaratively through SWRL. Our framework offers a constructivist learning approach through the presentation of the LKO's context to the learner based on domain ontology. Finally, it allows the standardization of the generated learning objects in SCORM and IMS-LD.
\end{abstract}

\section{Introduction}

Due to the requirements of today's economy, learning must be adapted to fulfill just-in-time and just-enough learning needs. One way to model these needs is the adoption of a competence-based learning approach. This approach enables to exploit competence models for assembling instructional units to help the learner master targeted skills and competencies. We employ the term "instructional unit" instead of "learning object" on purpose. In fact, learning objects are black-box structures that are not fine-grained enough to attain such learning goals. Moreover, most existing content takes the form of whole learning objects stored in Learning Object Repositories (LORs). Consequently, content must be refactored to match a competence model [1].

Text mining and semantic annotation could be used for this purpose. The extraction and annotation of implicit and significant information from learning resources through text mining fosters their reusability: they allow the indexing of learning resources according to their actual content and they provide a way to automatically aggregate useful learning resources based on their content. In this paper, we focus on text mining over textual learning objects. We do not deal with other formats (image, sound, etc.).

Another issue in competence-based models lies in the ability to efficiently sequence the retrieved content to better fulfill competence gaps [1]. The use of instructional design when sequencing learning content could be an answer to this need. In fact, the lack of an explicit pedagogical framework in the creation of learning objects leads to very poor capabilities in their automatic composition and effective reuse.

This paper presents an integrated approach for refactoring e-Learning resources based on a competence model. It is organized as follows. Section 2 presents the text mining and annotation processes involved in the creation of knowledge objects from textual documents. Section 3 presents the instructional patterns that we use to aggregate relevant learning knowledge objects. Section 4 and 5 talk about learning material deployment and standardization. Finally, section 6 provides a section about related work before a summary and concluding remarks.

\section{Knowledge Objects Creation through Text Mining and Annotation}

Competence-based learning models work by linking skills and competencies to the most appropriate learning resources. Semantic web and ontologies can be of great utility to implement the model.

We propose an ontology-based framework that models competencies as well as the learning resources. In this model, a competence is a set of skills linked to domain concepts and constitutes a learning objective [2]. We chose to declare the skills according to the Bloom Taxonomy [3] because it is a formal model which describes six levels of knowledge mastery (knowledge, comprehension, application, analysis, synthesis, and evaluation). Each level is related to a set of verbs that defines the skills that can be mastered at this level. For instance, "define" is related to the knowledge level whereas "describe" is linked to the comprehension level. The skills are mapped on instructional roles through SWRL (Semantic Web Rule Language) rules. Instructional roles, which are defined in the instructional 
role ontology (IRO), indicate the pedagogical purpose of portions of documents such as a "definition", an "introduction", or an "explanation".

Figure 1 summarizes the ontological model.

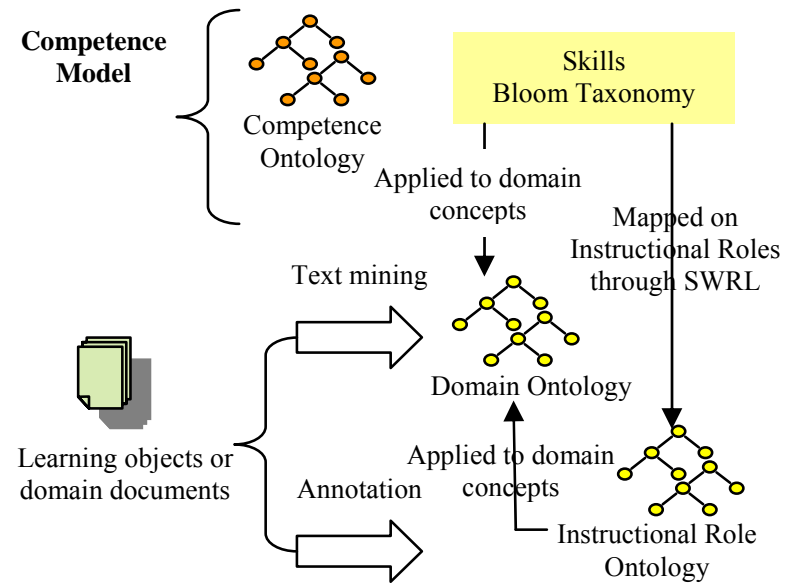

Figure 1 : The competence-based ontological model

Competence models require specific instructional units. Whole learning objects are not sufficiently finegrained to be put in context and re-purposed. This is why we believe that the vision of learning object repositories is not appropriate. Instead, we propose a knowledge object repository on which we can apply sequencing strategies. Knowledge objects are modular units that map to specific components of the competence model. The creation of these knowledge objects relies upon text mining and annotation.

\subsection{Text Mining}

Text mining deals with finding particular knowledge in learning objects through document parsing. It is applied on learning objects and domain documents to extract a domain ontology, which is linked to the competence model through semantic concepts. The domain ontology takes the form of a concept map composed of semantic concepts and relationships between them.

In order to determine domain concepts used in the competence-based model, linguistic analysis and machine learning algorithms are implemented to find potentially interesting concepts and relations between them. Such algorithms help identify, for example, significant semistructured information such as key phrases from documents. We use the Kea-3.0 algorithm [4] in order to determine the document key phrases. Then we collect the sentences that contain these key phrases and we parse them through a statistical natural language processing parser: the Stanford Parser [5]. We use its typeddependency parsing module [6] to extract grammatical relations between pairs of words (such as subject, object, etc). Then we perform a semantic analysis by applying lexico-semantic patterns over the grammatical relations. An example of pattern for defining a semantic concept is to assemble a word with its noun compound modifier. Another pattern for a semantic relation is to assemble a verb and its auxiliary, etc.

Figure 2 shows an example of the parsing process for the sentence "Learning content is composed of Assets that are electronic representations that are delivered to a Web client.". It is displayed through a Concept Map Generator and Editor. The first screenshot displays the grammatical concept map and the second one shows the resulting semantic map.

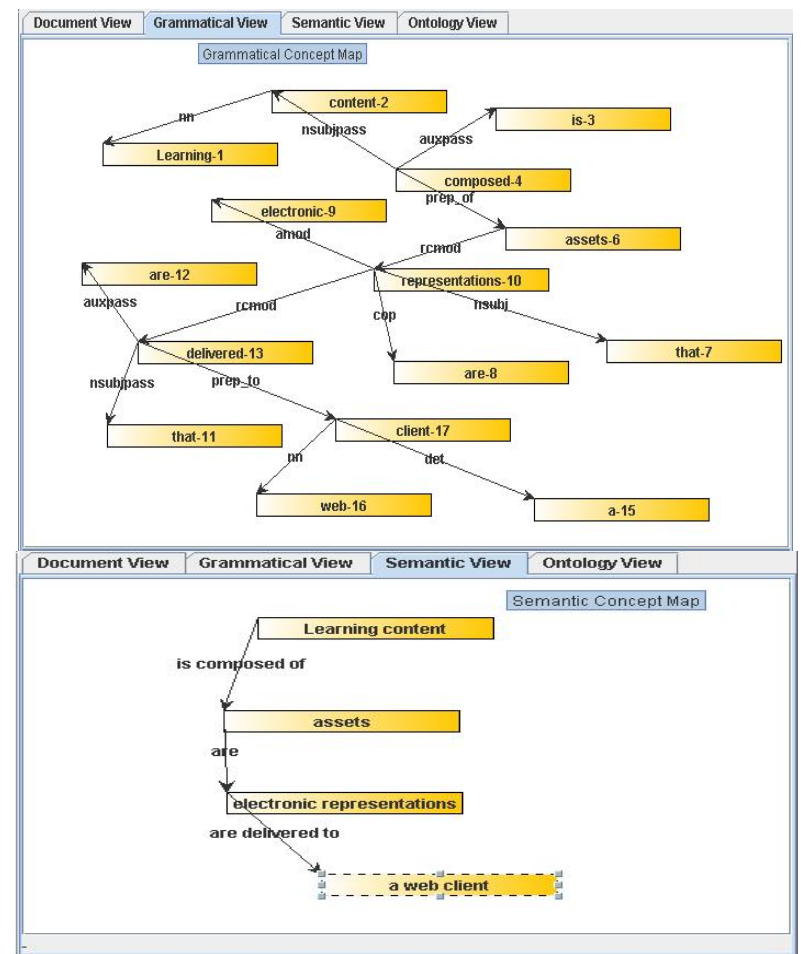

Figure 2 : The concept map generator and editor (grammatical view and semantic view)

For instance, the word "Content" has one noun compound modifier: "Learning". The resulting semantic concept is "Learning Content". The word "delivered" has an auxiliary ("are") and a subject ("that"). It is then translated into a semantic relationship "are delivered" between "electronic representations" and "Web client" and so on.

The resulting concept maps are stored in an OWL ontology (The domain ontology) that is used to index the learning objects and supports the competence model.

\subsection{Instructional Annotation}

Annotation is performed over learning objects to determine the instructional roles of their different sections. The Instructional Role Ontology represents the 
statement of a relationship between an instructional role and a domain concept. It is mapped on the competence ontology through SWRL rules. For instance, the skill "define" is associated to the instructional role "Definition", the skill "explain" to "Explanation" and so on.

The importance of modeling the relation between competencies and instructional roles through semantic rules instead of modeling it in the ontology relies on the ability to change them. A designer may decide that defining a concept requires an introduction, a definition and an example about the concept whereas another one may be satisfied with a sole definition.

In order to determine instructional roles in learning objects, annotation is used by directly mapping the learning object content to the instructional role ontology. This is performed manually through a Knowledge Annotator by simple drag and drops of document portions over the desired instructional role.

At the end of the mining and annotation processes, a learning object or a document is indexed according to domain and instructional roles ontologies. This enables a focused research of documents or portions of documents according to both dimensions (domain and instruction).

\section{Creating Instructional Patterns aggregate Learning Objects}

We evoked the challenges that face a competencebased learning model in the introduction. The second challenge involves instructional design in creating meaningful learning content. Instructional design theories try to implement the best design of instruction so that learning succeeds. Indeed, there are no instructional design elements related to sequencing learning objects within existing standard metadata [7]. Even if IMS-LD defines a pedagogical language for creating learning scenarios and refers to educational theories [8] (such as Gagné, Merrill, etc.), it does not exploit these theories to deploy learning material. In fact, a new trend in the elearning and intelligent tutoring system communities insists on the importance of using instructional theories. Bourdeau et al. [9] suggest that pedagogical knowledge should be encoded declaratively in order to make authoring systems theory-aware. Following this idea, our goal is to exploit the instructional roles in an instructional theory ontology through SWRL rules. The instructional theory is modeled as a set of instructional steps, which combines the various instructional roles in order to facilitate some piece of learning. We believe that the ability to follow an instructional strategy in sequencing knowledge objects is necessary to obtain effective LEARNING objects.

An Instructional Plan Generator is used to generate Learning Knowledge Objects (LKOs) compliant to the selected instructional theory. It indicates the available and missing instructional roles linked to each instructional step. The designer can add the missing roles, he can view the rules used at each step of the theory, or he can ask for the generation of the LKO according to another theory for comparison purposes.

For instance, we modeled the Gagné theory [10] as an instance of the class "InstructionalTheory". This theory is based on nine events of instruction (Gain learner's attention, inform the learner of expected outcomes, etc.). An example of rule using the Gagné theory for the instructional event: "Gain Attention" is, for instance, to present an illustration for the concept under study:

InstructionalStep(Gain_attention) And Concept(?y) And AssetCategoryType(?z) And assetCategory(?y, ?z) $\rightarrow$ Illustration(?z) And concept(?z,?y)

The on-the-fly generation of a learning knowledge object is not constrained by one theory as the LKO's structure (the theory's steps) is separated from the content (the instructional roles).

\section{Learning Material Deployment and Context Exploration}

Competence-based learning focuses on individual adaptation and avoids the presentation of previously mastered knowledge. The system investigates the learner model to adapt its behavior to the learner. We perform adaptation through a Competence Gap Analyzer that analyzes the differences between the required competence and the learner model.

A Learning Knowledge Object is generated for each skill in a competence according to a given instructional theory. It leads the learner to the mastery of predefined objectives stored in the competence ontology. The learner receives guidance by following the generated structure and the presentation of the LKO content.

The key issue in deploying learning objects effectively is to provide ways for the learner to put the information in context. The constructivist theory states that learning objects can be confusing or meaningless without context. Indeed, the significance of a concept is not absolute but is closely linked to its context. A Learning Knowledge Object possesses the context of the concept that it addresses expressed as a concept map taken from the domain ontology. This context is generated by assembling a concept map composed of the semantic representations of the knowledge objects referring to this concept. A Learning Knowledge Object offers an active individual exploration of its content to the learner through free navigation support and search facilities. It allows free navigation around the main concept context, adapted to the learner model. The learner can ask for the on-the-fly generation of a LKO about a related concept. His comprehension can be enhanced by the relationships between concepts by helping him to refer to prior knowledge. 


\section{Learning Objects Normalization}

The importance of e-Learning specifications in eLearning requires that any proprietary object has exporting mechanisms. Our goal is to produce learning knowledge objects that can be compatible with two specifications: the SCORM standard, which has achieved a wide adoption among the e-leaning community and the IMS-LD specification, which is also gaining importance.

\subsection{SCORM Normalization}

To achieve SCORM standardization, a Learning Knowledge Object is packaged as a content aggregation. Each generated Learning Knowledge Object corresponds to a skill that must be mastered. Each skill is formalized as a SCORM activity composed of multiple Sharable Content Objects (SCO) that represent each instructional step of the applied instructional theory. For the moment, we use a linear sequencing strategy (choice=true, choiceExit=true, flow=true) to teach each Learning Knowledge Object.

The instructional objective metadata for the activity is created under the LOM classification element with an "educational objective" purpose and the "discipline" purpose is employed to define the domain concept concerned by the described skill.

In order to test the learning knowledge objects compliance with the SCORM standard, we used The SCORM 2004 3rd Edition Conformance Test Suite. It contains the conformance testing software to perform self-testing on LMSs, SCOs and Content Packages. We ran two kinds of tests: a first test of the LKO structure against the SCORM Content Aggregation Content Package Application Profile, which succeeded. We also performed a second test by importing the learning knowledge Object structure into the SCORM Runtime Environment (Sample RTE 1.3.3). This second test succeeded also and we were able to launch the LKO in the Sample RTE 1.3.3 as shown in figure 3 .

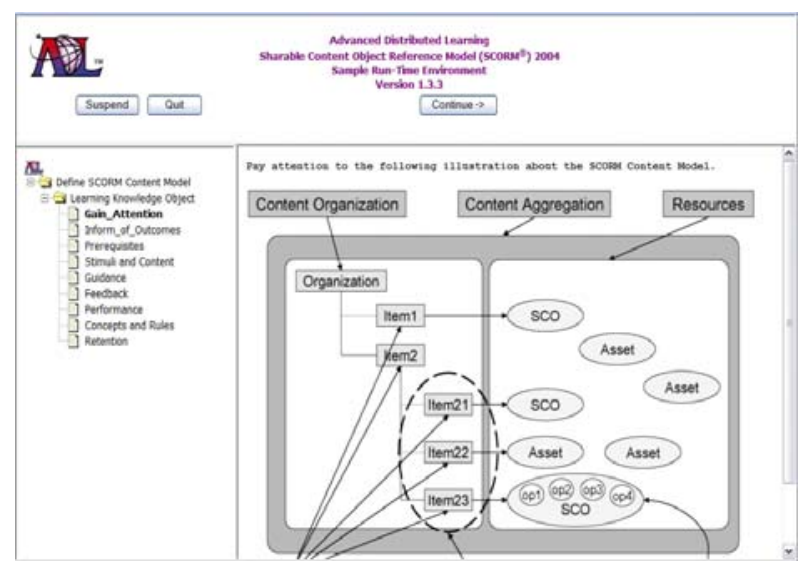

Figure 3: The preview of the LKO in the sample RTE 1.3.3.

\subsection{IMS-LD Normalization}

IMS-LD is a language for modeling units of learning. In IMS-LD, the structure of the learning scenario is separated from the learning materials and services. Like in the SCORM specification, a content package conformant to the IMS Content Packaging norm must be provided. It contains the manifest as well as a valid IMS learningdesign element and the required learning resources.

Competence skills are represented, in IMS-LD, as activity structures. Each step of the instructional theory that is used is linked to a learning activity. There are as many learning activities as there are instructional steps in the theory.

Figure 4 shows an IMS-LD Learning design manifest.

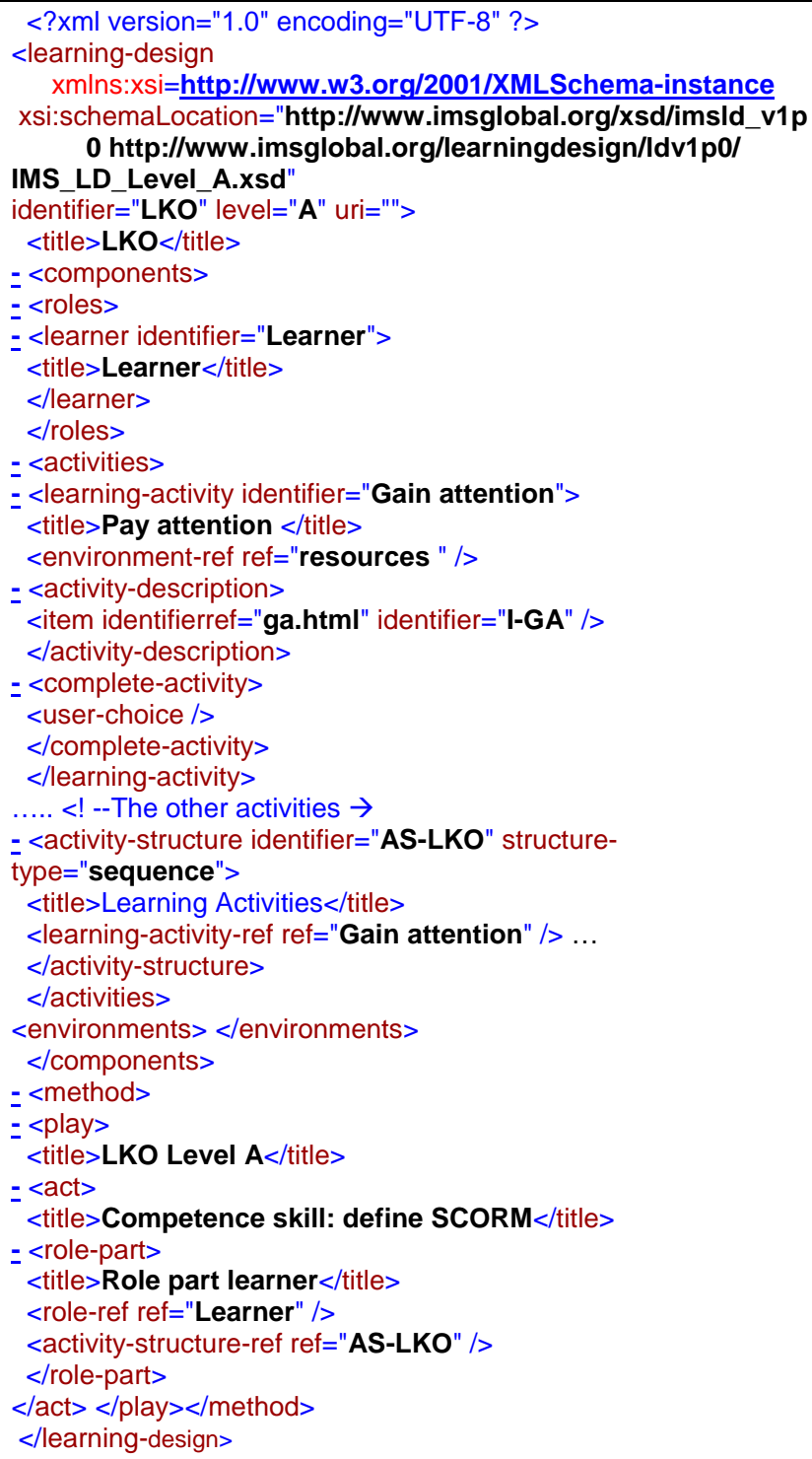

Figure 4: An IMS-LD Learning Design manifest 


\section{Related Works}

A number of learning content models already exist such as the SCORM content aggregation model, the AICC specification or the ALOCOM Model [11]. However, few of them support the semantic web technologies (Ontology-based content and metadata) and use it to sustain the definition of content objects. Furthermore the real semantic content is unknown as well as the instructional content of learning resources. For example, when considering a web page asset, the following questions remain unanswered with current content models: What is the content of the web page? What is the pedagogical design behind it? What is the instructional role of each of its component?

An educational metadata standard should indicate how to specify instructional aspects of a learning resource such as instructional theories and metadata. This could help to find learning resources for a "just-in-time, just-enough" learning aim more tailored to learner's needs and profile. Ontologies could help formalize such a framework. However, they are poorly represented in current content models and especially in most used e-Learning standards: SCORM and IMS-LD.

The new content model presented in this paper, the Knowledge Puzzle Content Model, presents a solution to the above issues.

\section{Conclusion}

Text mining can have a great impact on e-learning. It has been used, in the e-learning community, for classifying learner trends and patterns [12], and for extracting metadata automatically $[13,14]$. To the best of our knowledge, few research work paid attention to the generation of a domain ontology to index learning object content [15] or tackled the issue of using natural language processing to create domain concept maps. Few, if any, research related the generated domain ontology to a theoretically-sound competence-based model. Finally, none of them considered the declarative support of instructional theories to generate a pedagogically-explicit learning object and the standardization of the resulting learning objects.

In this paper, we presented an approach to generate dynamically learning knowledge objects (LKOs) based on a competence model and guided by instructional theories. We used various ontologies to structure its content. We also showed that we were able to export such LKOs into standard formats (SCORM, IMS-LD). Further research might annotate instructional roles automatically and evaluate more thoroughly the generated domain concept maps.

\section{References}

[1] Tuso, G. and Longmire, W. "Competency-Based Systems and the Delivery of Learning Content", , in Brightman, D. (Eds.), Learning Without Limits, Vol. 3, pp. 33-38, Informania Inc, 2000.

[2] Nkambou, R., Frasson, C., and Gauthier, G. "CREAMTools: An Authoring Environment for Knowledge Engineering in Intelligent Tutoring Systems", in Authoring Tools for Advanced Technology Learning Environments: Toward costeffective, adaptative, interactive, and intelligent educational software, pp. 93-138, Kluwer Publishers, 2003.

[3] Bloom, B. S. (1956). Taxonomy of educational objectives: The classification of educational goals: Handbook I, cognitive domain, Longman, New York.

[4] Frank, E., Paynter, G.W., Witten, I..H., Gutwin, C., and Nevill-Manning, C.G.. Domain-specific key phrase extraction, in Proc. of the 16th International Joint Conference on Artificial Intelligence, pp. 668-673, San Francisco, 1999.

[5] Klein, D. and Manning, C.D. Accurate unlexicalized parsing, in Proc. of the 41st Meeting of the Association for Computational Linguistics, pp. 423 - 430, Sapporo, Japan, 2003. [6] De Marneffe, M-C., MacCartney, B. and Manning, C.D. Generating Typed Dependency Parses from Phrase Structure Parses, in Proc. of 5th Conference on Language Resources and Evaluation, Genoa, Italy, 2006.

[7] Wiley, D.A. "Connecting learning objects to instructional design theory: a definition, a metaphor, and a taxonomy", in Wiley, D. (Ed.), The instructional use of learning objects: 2000. [8] Psyché, V., Bourdeau, J., Nkambou, R. and Mizoguchi, R. Making Learning Design Standards Work with an Ontology of Educational Theories, in Proc. of the 12th Artificial Intelligence in Education, pp. 539-546, Amsterdam, The Netherlands, 2005.

[9] Bourdeau, J., Mizoguchi, R., Psyché, V., and Nkambou, R. Selecting Theories in an Ontology-Based ITS Authoring Environment, in Proc. of Intelligent Tutoring Systems, pp.150161, Maceiò, Brazil, 2004.

[10] Gagné, R. M., Briggs, L. J., and Wagner, W. W. Principles of Instructional Design (4th Ed.), HBJ College Publishers, Fort Worth, 1992.

[11] Verbert, K. and Duval, E. Towards a Global Architecture for Learning Objects: A Comparative Analysis of Learning Object Content Models, in Proc. of World Conference on Educational Multimedia, Hypermedia and Telecommunications, pp. 202-208, Chesapeake, VA, 2004.

[12] Desikan, P., DeLong, C. Beemanapalli, K. Bose, A. and Srivastava, J. "Web Mining For Self Directed E-Learning", in Romero, C. and Ventura, S. (Eds.): Data Mining for E-Learning, pp. 21-37, WIT Press, 2005.

[13] Cardinaels K., Meire M., and Duval E. Automating Metadata Generation: the Simple Indexing In-terface, in Proc. of the 14th international conference on World Wide Web, pp.548556, Chiba, Japan, 2005.

[14] Jovanović, J., Gasevic, D., and Devedzic, V. "Ontologybased Automatic Annotation of Learning Content", International Journal on Semantic Web and Information Systems, 2(2): 91119, 2006.

[15] Gasevic, D., Jovanovic, J., Devedzic, V. Ontologies for Creating Learning Object Content, in Proc. of the 8th International Conference on Knowledge-Based Intelligent Information \& Engineering Systems, pp. 284-291, Wellington, New Zealand, 2004. 